\title{
Naming the Mini-Gastric Bypass
}

\section{Robert Rutledge}

Published online: 23 September 2014

(C) Springer Science+Business Media New York 2014

To the Editor:

I am pleased to comment on the suggestions to rename the operation which I named the "Mini-Gastric Bypass" in 1997. Publications from around the world demonstrate that my initially good results are confirmed. This is particularly rewarding given the early criticism attended to the mini-gastric bypass (MGB) in its early years. Critics said that the pouch was too big, the anastomosis too large, and there would be devastating and unmanageable bile reflux gastritis and that there be no weight loss. They were incorrect.

Carbajo and Caballero modified the MGB adding an "antireflux" technique and called their version the "One-Anastomosis Gastric Bypass (OAGB)". Others have suggested the Omega Loop Gastric Bypass which is a suitable name, but has its own limitations. The long-used names MGB and OAGB thus stand, and the use of Single Anastomosis Gastric Bypass (SAGB) is similar to OAGB and is likely to be confused with the various SADI procedures.

The MGB is restrictive while not being obstructive. In contrast to the small pouch and small gastrojejunostomy of the RYGB, the tight gastric pouch of the sleeve, and the fixed plastic of the Lap-band, the MGB uses a larger gastric pouch with a wide open gastrojejunostomy to allow rapid emptying into the jejunum.

Also, in contrast to the RYGB, the MGB has a significant malabsorptive component. It induces significant fatty food intolerance, an increase in bowel movements, and mild steatorrhea in response to large fatty meals. The operation has been found to induce a "Mediterranean-type" diet postoperatively with a decrease in intake of sugar-sweetened beverages, fatty foods, processed meats, and an increase in yogurt, fresh fruits, and vegetables.

I believe now that my initial findings have been well confirmed; the MGB/OAGB is a short simple operation that is safe in the short and long terms. MGB/OAGB provides one of the best and most durable weight loss of any bariatric operation; it can be easily tailored to treat the spectrum of metabolic disease from the thin diabetic to the super-super obese, and it can easily be reversed or revised.
R. Rutledge $(\bowtie)$

Center of Laparoscopic Obesity Surgery, 1000 North Green Valley

Parkway, Suite 440 box 492, Henderson, NV 89074, USA

e-mail: drr@clos.net 